\title{
ANTIBACTERIAL ACTIVITY OF HYDROXYIMIDAZOLE DERIVATIVES
}

\author{
Suaad M. Abuskhuna*1, Talal H. Zeglam¹, Omran N. R. Fhid ${ }^{1}$, Asma O. Jebril ${ }^{1}$ \\ 1*Department of Medicinal and Pharmaceutical Chemistry, Faculty of Pharmacy, University of Tripoli, Tripoli, \\ Libya.
}

\section{ABSTRACT}

The imidazole ring is containing one of the important heterocyclic compounds found in endogenous biomolecules and pharmaceutical preparations. Different methods have been reported for the synthesis of 1-Hydroxyimidazoles and a number of them reported to possess significant biological activity, thus synthesis of some new derivatives are introduced for better antibacterial activity.

\section{KEYWORDS}

Imidazole, Hydroxyimidazole derivatives and Antibacterial activity.

\section{Author for Correspondence:}

Suaad M. Abuskhuna,

Department of Medicinal and Pharmaceutical

Chemistry, Faculty of Pharmacy,

University of Tripoli, Tripoli, Libya.

Email: sudskhun@hotmail.com

Available online: www.uptodateresearchpublication.com

\section{INTRODUCTION}

The imidazole ring is containing five membered ring systems have been described for their biological activity against various micro $\operatorname{organisms}^{1,2}$. It is commonly found as a part of structure substances such as histamine and purine, also in a number of pharmaceutical drugs, such as antifungal agent ${ }^{1}$ (ketoconazole), antiprotozoal agentsmetronidazole $^{3}$ (1) and antihistamine cimetidine $^{4}(2) . \mathrm{N}$-hydroxyimidazoles are analogous of imidazole, have been prepared and tested biologically. Besides this, apesticidal screening was carried out by Allan $^{5}$ on a series of $N$ hydroxyimidazoles (3) and found them to have herbicide and insecticide activities. Antihypertensive ${ }^{6}$ activity was observed in some

January - March 
$N$-hydroxyimidazole-5-methanamine derivatives such as (4). (Figure No.1). They have been also applied to coloring materials ${ }^{7}$.

1-Hydroxyimidazoles were prepared by condensation reaction of monoximes of 1,2 diketones with an aldehyde in presence of ammonia $^{5}$ (Scheme No.1). They were prepared by reduction of 3-hydroxy-imidazole-1-oxide, and by cyclization of a 1,2-diketone, and an aldehyde in the presence ofhydroxyl-amine ${ }^{7,8}$. $\mathrm{N}$-hydroxyimidazole has also been synthesized by $\mathrm{N}$-oxidation of imidazole with 3-chloroperbenzoic $\operatorname{acid}^{7}$ or peroxyphethalic acid ${ }^{8,9}$. The compounds in the present work have been synthesized by using Akagane method $^{7}$ (Table No.1). The reproduced compounds and the new derivatives $(10,11)$ were identified by means of physical and spectrophotometric analysis and evaluated in vitro for their antimicrobial activity.

\section{MATERIAL AND METHODS}

All solvents and reagents were used as received from various vendors. Infrared spectra were recorded by using $\mathrm{KBr}$ disc in the region 4000$400 \mathrm{~cm}^{-1}$ on a Nicolet FT-IR Impact 400D infrared spectrometer. ${ }^{1} \mathrm{H}$ NMR and ${ }^{13} \mathrm{C}$ NMR spectra were run as solutions in $\mathrm{CDCl}_{3}$ or DMSO- $\mathrm{d}_{6}$ on a Bruker Advance $300 \mathrm{MHz}$ instrument. Mass spectra were carried out on a Kratos Profile mass spectrometer. The melting points were determined by open capillary method and were uncorrected.

General procedure for preparation of $1 \mathrm{H}$ imidazole-1-ol 4, 5-Dimethyl-2-(pyridine-2-yl)-1Himidazole-1-ol ${ }^{5}(5)$

The general procedure of Akagane and coworkers was used. A $250-\mathrm{mL}$, round-bottomed flask, equipped with a magnetic stirrer was charged with diacethylmonoxime $(1.5 \mathrm{~g}, 15 \mathrm{mmol})$ dissolved in a mixture of ammonia and water (1:1). Picolinaldehyde $(1.5 \mathrm{~g}, 14 \mathrm{mmol})$ was added and the mixture was stirred overnight a room temperature. The solvent was removed under reduced pressure and the product was extracted with chloroform. The chloroform extract was dried over magnesium sulphate, filtered and evaporated under vacuum.

Available online: www.uptodateresearchpublication.com
The residue was crystallised from toluene to give (1) as yellow crystals $(1 \mathrm{~g}, 30 \%), \mathrm{mp}=139-141^{\circ} \mathrm{C}$.

${ }^{1} \mathrm{H} \mathrm{NMR}\left(\mathrm{CDCl}_{3}\right): 2.22(6 \mathrm{H}, \mathrm{s}), 7.21-7.16(1 \mathrm{H}, \mathrm{m})$, 7.82-7.76 $(1 \mathrm{H}, \mathrm{dt}, \mathrm{J}=7.8,1.7 \mathrm{~Hz}), 8.19(1 \mathrm{H}, \mathrm{s})$, $8.38(1 \mathrm{H}, \mathrm{d}, \mathrm{J}=7.5 \mathrm{~Hz})$.

${ }^{13} \mathrm{C}$ NMR $\left(\mathrm{CDCl}_{3}\right): 7.6,13.0,119.7,122.2,122.8$, $131.0,138.4,146.5,149.3$.

IR (KBr): 3382, 2421，1639, 1588, 1524, 1488, 1310, 1231, 1154, 1115, 964, 794, 742, 610, 505 $\mathrm{cm}^{-1}$.

m/z: $189\left(M^{+}, 16 \%\right), 172(64), 105$ (56), 95 (8), 79 (100), 69 (28), 51 (58).

\section{4, 5-Diphenyl-2-(pyridine-2-yl)-1H-imidazole-1-ol ${ }^{5}$}

(6)

The product was prepared in a similar method to (1) using $\alpha$-benzilmonoxime $(2 \mathrm{~g}, \quad 8.8 \mathrm{mmol})$ and picolinaldehyde $(1 \mathrm{~g}, 9 \mathrm{mmol})$. The product was crystallised from toluene giving (2) as yellow crystals $(2.5 \mathrm{~g}, 89 \%), \mathrm{mp}=163-164^{\circ} \mathrm{C}$.

${ }^{1} \mathrm{H}$ NMR $\left(\mathrm{CDCl}_{3}\right)$ : 7.30-7.19 (4H, m), 7.52-7.38 $(3 \mathrm{H}, \mathrm{m}), 7.61-7.56(4 \mathrm{H}, \mathrm{m}), 7.92-7.86(1 \mathrm{H}, \mathrm{dt}, \mathrm{J}=$ $7.8 \mathrm{~Hz}), 8.27(1 \mathrm{H}, \mathrm{d}, \mathrm{J}=8 \mathrm{~Hz}), 8.44(1 \mathrm{H}, \mathrm{d}, \mathrm{J}=5$ $\mathrm{Hz})$.

${ }^{13} \mathrm{C}$ NMR $\left(\mathrm{CDCl}_{3}\right): 1202,122.5,125.8,126.9$, $127.4,128.2$, 128.30, 128.37, 128.4, 129.9, 132.2, 134.6, 134.9, 138.4, 145.7, 149.8.

IR (KBr): 3063, 1606, 1562, 1531, 1476, 1439, $1402,1309,1148,959,783,730,700 \mathrm{~cm}^{-1}$.

m/z: $313\left(\mathrm{M}^{+}, 26 \%\right), 296$ (28), 284 (18), 193 (22), 176 (8), 165 (36), 148 (14), 105 (58), 89 (58), 79 (92), 63 (54), 51 (100).

4, 5-Dimethyl-1H, 3'H-[2, 4'-biimidazol]-1-ol ${ }^{10}$ (7) The white solid was prepared in a similar method to (1) using diacethylmonoxime $(0.5 \mathrm{~g}, 5 \mathrm{mmol})$ and 4 (5)-imidazole carboxaldehyde $(0.5 \mathrm{~g}, 5 \mathrm{mmol})$. The solid was crystallised from ethyl acetate to afford (3) as white crystals $(0.75 \mathrm{~g}, 80 \%), \mathrm{mp} 155^{\circ} \mathrm{C}$.

${ }^{1} \mathrm{H}$ NMR (DMSO-d $\left.\mathrm{d}_{6}\right): 1.91(3 \mathrm{H}, \mathrm{s}), 2.01(3 \mathrm{H}, \mathrm{s})$, $7.64(2 \mathrm{H}, \mathrm{s})$.

${ }^{13} \mathrm{C}$ NMR (DMSO- $\mathrm{d}_{6}$ ): 7.2, 10.9, 118.5, 121.9, 123.2, 126.2, 131.3, 135.9.

IR (KBr): 3079, 1646, 1461, 1295, 1221, 1172, 1129, 1086, 1006, 870, 704, 633 $\mathrm{cm}^{-1}$.

m/z: 178 ( $\left.M^{+}, 28 \%\right), 161$ (82), 147 (4), 120 (18), 94 (100), 81 (10), 68 (66), 64 (26), 60 (10), 55 (48).

January - March 
Suaad M. Abuskhuna. et al. / Asian Journal of Pharmaceutical Analysis and Medicinal Chemistry. 8(1), 2020, 1-6.

\section{2-(2, 4-Dimethoxyphenyl)-4, 5-diphenyl-1H-} imidazol-1-ol ${ }^{11}(8)$

This solid was prepared in a similar method of (1) using 2, 4-dimethoxybenzaldehyde $\quad(1.47 \mathrm{~g}$, $8.84 \mathrm{mmol})$ and $\alpha$-benzilmonoxime $(2.0 \mathrm{~g}$, $8.88 \mathrm{mmol})$. The product was crystallised from toluene giving a white solid $(2.1 \mathrm{~g}, 63 \%), \mathrm{mp} 196-$ $197^{\circ} \mathrm{C}$.

${ }^{1} \mathrm{H} \mathrm{NMR}\left(\mathrm{CDCl}_{3}\right): 3.75(3 \mathrm{H}, \mathrm{s}), 3.85(3 \mathrm{H}, \mathrm{s}), 6.50-$ $6.47(2 \mathrm{H}, \mathrm{d}, \mathrm{J}=8.9 \mathrm{~Hz}), 7.38-7.25(11 \mathrm{H}, \mathrm{m})$.

${ }^{13} \mathrm{C}$ NMR $\left(\mathrm{CDCl}_{3}\right): 27.3,55.8,56.55,99.3,105.6$, 127.3, 128.5, 129.4, 130.7, 162.0.

IR (KBr): 3413, 3070, 2943, 2845, 1619, 1585, 1541, 1468, 1302, 1214, 1165, 1136, 1034, 973, $919,835,804,764,701,654,517 \mathrm{~cm}^{-1}$.

m/z: $372\left(M^{+}, 10 \%\right), 355$ (18), 178 (1), 165 (2), 103 (4), 91 (100), 84 (26), 77 (6), 65 (14), 56 (36).

2-(2-Hydroxyphenyl)-4, 5-dimethyl-1H-imidazol-1$o l^{5}(9)$

This solid was prepared in a similar way to (1) using diacetylmonoxime $(1.0 \mathrm{~g}, 10 \mathrm{mmol})$ and salicyladehyde $(1.2 \mathrm{~g}, 10 \mathrm{mmol})$. The solid was crystallised from ethanol/acetonitrile giving colourless crystals $(1.3 \mathrm{~g}, 64 \%), \mathrm{mp} 245^{\circ} \mathrm{C}$.

${ }^{1} \mathrm{H}$ NMR (DMSO-d $\left.\mathrm{d}_{6}\right): 2.10(3 \mathrm{H}, \mathrm{s}), 2.20(3 \mathrm{H}, \mathrm{s})$, 6.82-6.88 $(2 \mathrm{H}, \mathrm{m}), 7.27-7.33(1 \mathrm{H}, \mathrm{dt}, \mathrm{J}=7.7 \mathrm{~Hz})$, $7.46(1 \mathrm{H}, \mathrm{d}, \mathrm{J}=7.5 \mathrm{~Hz})$.

${ }^{13} \mathrm{C}$ NMR (DMSO- $\mathrm{d}_{6}$ ): $7.25,10.0,113.3,118.4$, 119.8, 122.5, 124.1, 127.6, 131.7, 134.2, 158.6.

IR (KBr): 2927, 1646, 1609, 1436, 1307，1276, $1166,972,910,849,753,541 \mathrm{~cm}^{-1}$.

m/z: $204\left(M^{+}, 60 \%\right), 187(100), 159(68), 145$ (16), 121 (40), 105 (16), 91 (14), 80 (20), 77 (38), 68 (28), 51 (52).

\section{4, 5-Dimethyl-2-[(E)-2-phenylvinyl]-1H-imidazol- 1-ol (10)}

The yellow solid was prepared in a similar way to (1) using cinnamaldehyde $(0.26 \mathrm{~g}, 1.96 \mathrm{mmol})$ and diacetylmonoxime $(0.2 \mathrm{~g}, 1.97 \mathrm{mmol})$. The solid was crystallised from acetonitrile/ethanol giving yellow crystals $(0.36 \mathrm{~g}, 85 \%), \mathrm{mp} 94-95^{\circ} \mathrm{C}$.

${ }^{1} \mathrm{H}$ NMR (DMSO-d $)$ ): $1.82(6 \mathrm{H}, \mathrm{s}), 6.73(1 \mathrm{H}, \mathrm{d}, \mathrm{J}=$ $16.4 \mathrm{~Hz}), 7.05(2 \mathrm{H}, \mathrm{m}), 7.14(2 \mathrm{H}, \mathrm{t}, \mathrm{J}=7.4 \mathrm{~Hz})$, $7.26(2 \mathrm{H}, \mathrm{d}, \mathrm{J}=7.4 \mathrm{~Hz})$.

Available online: www.uptodateresearchpublication.com
${ }^{13} \mathrm{C}$ NMR (DMSO- $\mathrm{d}_{6}$ ): 7.6, 126.6, 128.1, 128.2, 129.1, 136.9, 162.8.

IR (KBr): 3035, 1648, 1477, 1320, 1199, 1138, 1024, 966, 891, 748, 689, 561, cm ${ }^{-1} . \mathrm{m} / \mathrm{z}: 214\left(M^{+}\right.$, 34\%), 197 (100), 182 (26), 128 (26), 115 (42), 103 (30), 98 (12), 89 (14), 77 (42), 68 (12), 63 (26), 58 (14), 51 (42).

4, 5-Bis(4-fluorophenyl)-2-pyridin-2-yl-1Himidazol-1-ol (11)

This solid was prepared in a similar way to (1) using pyridine-2-carboxaldehyde $(0.15 \mathrm{~g}, 1.40 \mathrm{mmol})$ and $4, \quad 4^{\prime}$-difluorobenzilmonoxime $\quad(0.24 \mathrm{~g}$, $0.91 \mathrm{mmol})$. The solid was recrystallised from cyclohexane/ethylacetate giving yellow crystals (0.3g, 74\%), mp $163-164^{\circ} \mathrm{C}$.

${ }^{1} \mathrm{H}$ NMR $\left(\mathrm{CDCl}_{3}\right): 6.99(2 \mathrm{H}, \mathrm{t}, \mathrm{J}=8.7 \mathrm{~Hz}), 7.13$ $(2 \mathrm{H}, \mathrm{t}, \mathrm{J}=8.7 \mathrm{~Hz}), 7.33(1 \mathrm{H}, \mathrm{t}, \mathrm{J}=6 \mathrm{~Hz}), 7.51-7.56$ $(4 \mathrm{H}, \mathrm{m}), 7.92(1 \mathrm{H}, \mathrm{t}, \mathrm{J}=9 \mathrm{~Hz}), 8.24(1 \mathrm{H}, \mathrm{d}, \mathrm{J}=8$ $\mathrm{Hz}), 8.46(1 \mathrm{H}, \mathrm{d}, \mathrm{J}=5 \mathrm{~Hz})$.

${ }^{13} \mathrm{C}$ NMR $\left(\mathrm{CDCl}_{3}\right): 115.1,115.4,115.6,115.9$, $120.2,122.7,124.0,124.1,124.6,129.0,129.1$, $130.6,131.6,131.7,132.2,134.1,138.5,145.7$, 149.6, 160.4, 161.0163.7, 164.3.

IR (KBr): 3075, 1603, 1516, 1480, 1393, 1233, 1160, 1092, 956, 836, 815, 778, 735, 670, 578 $\mathrm{cm}^{-1}$. $\mathrm{m} / \mathrm{z}: 349$ (M+, 44\%), 332 (22), 211 (28), 201 (12), 150 (10), 123 (20), 107 (48), 95 (28), 79 (100), 69 (22), 57 (58).

4, 5-Dimethyl-2-(4-nitrophenyl)-1H-imidazole-1$o l^{5}(12)$

The yellow solid was prepared in a similar way to (1) using $p$-nitrobenzaldehyde $(1.0 \mathrm{~g}, 6.6 \mathrm{mmol})$ and diacetylmonoxime $(1.0 \mathrm{~g}, 10 \mathrm{mmol})$. The solid was crystallised from acetonitrile/ ethanol giving yellow crystals $(1.2 \mathrm{~g}, 55 \%), \mathrm{mp} 221^{\circ} \mathrm{C}$.

${ }^{1} \mathrm{H}$ NMR (CDCl $): 2.25(3 \mathrm{H}, \mathrm{s}), 2.55(3 \mathrm{H}, \mathrm{s}), 8.05$ $(2 \mathrm{H}, \mathrm{d}, \mathrm{J}=9.1 \mathrm{~Hz}), 8.27(2 \mathrm{H}, \mathrm{d}, \mathrm{J}=9.1 \mathrm{~Hz})$.

IR (KBr): 3325, 2420, 1633, 1600, 1544, 1355, $1310,1231,1154,1115,855 \mathrm{~cm}^{-1}$.

\section{RESULTS AND DISCUSSION}

The Akagane ${ }^{7}$ method was followed for the synthesis of new derivatives $(10,11)$ and other hydroxyimidazole compounds. diacethylmonoxime or benzilmonoxime was reacted

January - March 3 
with an appropriate aldehyde as shown in Scheme No.1. The reaction was carried out in aqueous media using ammonia and water mixture $(1: 1)$ in a stoppard flask at room temperature. The products are formed in solid form with good yields. The reactions were monitored by TLC, using silica gel as an adsorbent and ethyl acetate-hexane in different ratios as eluent, and they were characterized by spectroscopic techniques (Table No.1).

\section{Biological Evaluation}

The antimicrobial activities were determined using agar-cup method by measuring the zone of inhibition in $\mathrm{mm}$. All newly synthesized compounds were screened in vitro for their antibacterial activity against gram positive bacteria such as B.sub: bacillus subtilis, St.aur: Staphylococcus aureus, MRSA: Metacillin-Resistant Staphylococcus Aureus and gram negative bacteria such as K.P: Klebsiellapneumoniae, E.Coli: Escherichia coli, P.aeru: Pseudomonas aeruginosa by agar plate method. Nitrofurantoin $(\mathrm{F}, 300 \mu \mathrm{g})$ is a standard reference antibiotic used for antibacterial comparison activity with tested synthesized compounds (5-12, Table No.1).
DMSO 5\% was used as solvent to enhance the solubility of the compounds. The zone of inhibition was recorded in $\mathrm{mm}$ after incubation of plates of agar medium for $24 \mathrm{hrs}$ at $37^{\circ} \mathrm{C}$. The results of antibacterial activity indicated that compounds 10 , 12 have a good antibacterial activity compared to the standard drug as shown in Table No.2. Compound 10 has moderate activity on grampositive bacteria and have similar activity with same concentration on P. aeru and B. sub compared to nitrofurantoin, while compound 12 has exhibited good activity on St.aur and MRSA and showed no effect on the other species. The results did not show any antibacterial activity of the other prepared compounds (Table No.2). Structurally the imidazole ring of the prepared compounds is multi-substituted, these substituents make the ring unable to move freely and bind with function groups of bacterial proteins. The activity of compound 10 on some species of gram positive and gram negative may due to the double bond and phenyl group at position two. Substitution of phenyl group at para-position with nitro group provides compound 12 some moderate activity compared with compound 9 .

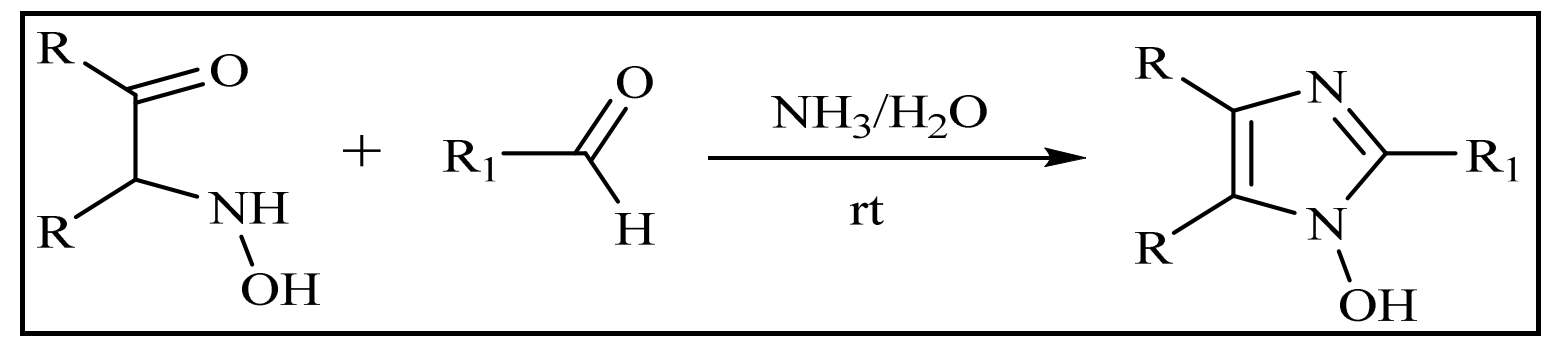

Scheme No.1: General synthetic reaction for 1-hydroxyimidazole derivatives Table No.1: Products of 1-Hydroxyimidazole derivatives

\begin{tabular}{|c|c|c|c|c|c|c|}
\hline S.No & Entry & $\mathbf{R}$ & $\mathbf{R}_{1}$ & Entry & $\mathbf{R}$ & $\mathbf{R}_{1}$ \\
\hline 1 & 5 & $\mathrm{CH}_{3}$ & & 9 & $\mathrm{CH}_{3}$ & \\
\hline 2 & 6 & $\mathrm{Ph}$ & & 10 & $\mathrm{CH}_{3}$ & \\
\hline 3 & 7 & $\mathrm{CH}_{3}$ & & 11 & & \\
\hline 4 & 8 & $\mathrm{Ph}$ & & 12 & $\mathrm{CH}_{3}$ & \\
\hline
\end{tabular}


Suaad M. Abuskhuna. et al. / Asian Journal of Pharmaceutical Analysis and Medicinal Chemistry. 8(1), 2020, 1-6.

Table No.2: Antibacterial activity of tested compounds 5-12

\begin{tabular}{|c|c|c|c|c|c|c|c|}
\hline \multirow{3}{*}{ S.No } & & \multicolumn{6}{|c|}{ Zone of inhibition (mm) } \\
\hline & & \multicolumn{3}{|c|}{ Gram negative bacteria } & \multicolumn{3}{|c|}{ Gram positive bacteria } \\
\hline & Compound & K.p & E. coli & P.aeru & B.sub & St.aur & MRSA \\
\hline 1 & 5 & 6 & 6 & 6 & 6 & 9 & 6 \\
\hline 2 & 6 & 6 & 6 & 6 & 6 & 6 & 6 \\
\hline 3 & 7 & 6 & 6 & 6 & 6 & 6 & 6 \\
\hline 4 & 8 & 6 & 6 & 6 & 6 & 6 & 6 \\
\hline 5 & 9 & 6 & 6 & 6 & 6 & 6 & 6 \\
\hline 6 & 10 & 6 & 6 & 11 & 12 & 14 & 14 \\
\hline 7 & 11 & 6 & 6 & 6 & 6 & 6 & 6 \\
\hline 8 & 12 & 6 & 6 & 6 & 6 & 12 & 13 \\
\hline 9 & DMSO & 6 & 6 & 6 & 6 & 6 & 6 \\
\hline 10 & $\mathrm{~F}$ & 18 & 18 & 11 & 11 & 20 & 21 \\
\hline
\end{tabular}

The concentration of each tested compounds and nitrofurantoin $\mathrm{F}$ is $300 \mu \mathrm{g}$

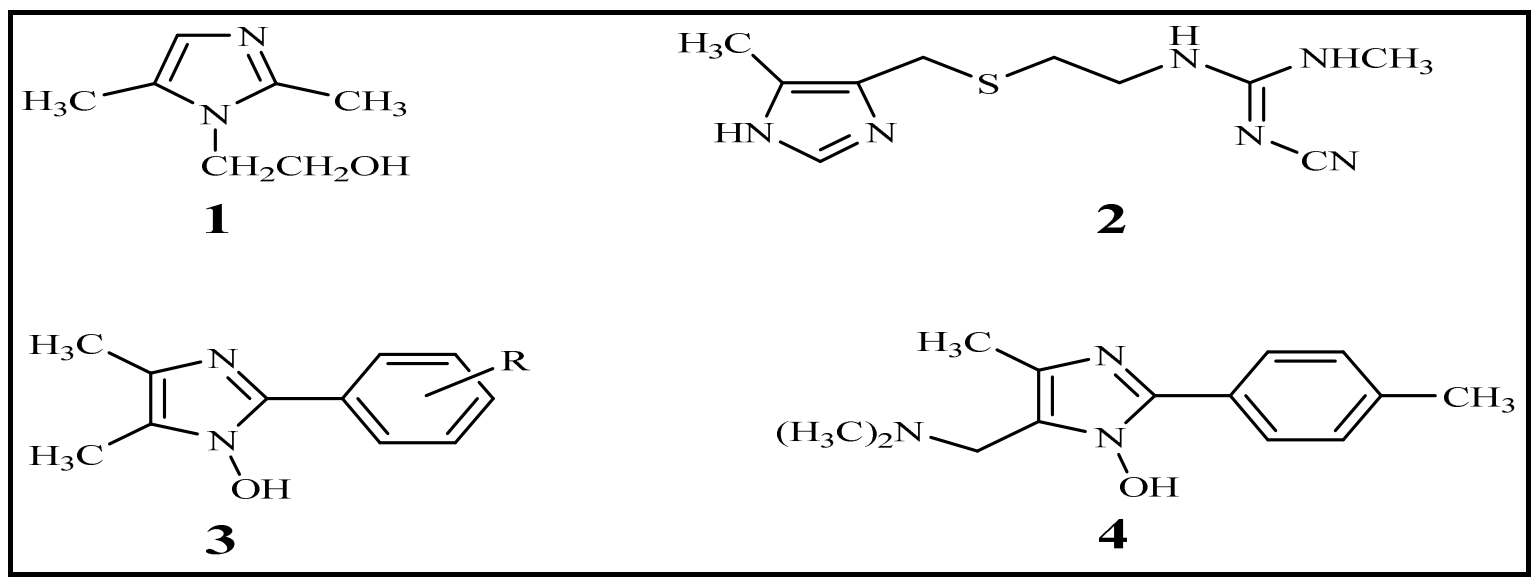

Figure No.1: Biologically active imidazole derivative

\section{CONCLUSION}

In general most of the prepared 1-hydroxyimidazole compounds have low or no activity against different species of bacteria, therefore, some structural modifications on imidazole ring are necessary to improve their activity.

\section{ACKNOWLEDGEMENT}

The author gratefully acknowledgement the technical support, and valuable suggestions obtained from microbiological Department.

\section{CONFLICT OF INTEREST}

We declare that we have no conflict of interest.
BIBLIOGRAPHY

1. Jain R, Jain S, Gupta R C, Anand N, Dutta G P, Puri S K. Indian J. Chem, 338, 1994, 251.

2. Mohamed A. Abd-Alla, Abdel-Hamid N. Ahmed, Maher F. El-Zohry and Farghaly A. Omar. Synthesis and Antibacterial Activity of Certain Quinoline Derivatives, Czech. Chem. Commun, 57(7), 1992, 1547.

3. Aldridge K E, Ashcraft D, O'Brien M and Sanders C V. Bacteremia due to Bacteroides fragilis group: distribution of species, $\beta$ lactamase production, and antimicrobial susceptibility patterns, Antimicrobial Agents and Chemotherapy, 47(1), 2003, 148-153. 
4. Larry E. Millikan. Drug Therapy in Dermatology, CRC Press, Marcel Dekker Inc, ISBN 978-0-203-90831-0. 2000, 82.

5. Allan G G, Chopra $\mathrm{C} S$ and Mattila $\mathrm{T}$. Imidazoles III. Pesticidal screening of some substituted 1-hydroxyimidazoles, Pest. Sci, 3(2), 1972, 153-159.

6. Philipp H A and Jirkovsky L I. American Home Products Crop, USA, 1980, 6.

7. Akagane $\mathrm{K}$ and Allan G G. Application of Imidazolic Compounds to Colour Materials (III), Shikizai Kyokaishi, 46(10), 1973, 560565.

8. Laus G, Stadlwieser J and Klotzer W. 1Hydroxyimidazole derivatives. III: Synthesis of 1-alkyloxy-, 1-arylalkyloxy-, and 1phenoxy-1H-imidazoles, Synthesis (Stuttgart), Thieme, New York, 10, 1989, 773-775.

9. Begtrup $\mathrm{M}$ and Vedso P. Preparation of Nhydroxyazoles by oxidation of azoles, $J$. Chem. Soc., Perkin Trans, 1(3), 1995, 243247.

10. Baus U and Reuther W. Eur. Pat. Appl. EP, 1991, 420. Chem. Abstr, 115, 1991, 29334r.

11. Eriksen B L, Vedso P, Morel S and Begtrup M. Synthesis of 2-Substituted 1Hydroxyimidazoles through Directed Lithiation, J. Org. Chem, 63(1), 1998, 12-16.

12. Thomas L. Lemke, David A. Williams, Victoria R, and Willaim Zito S. Foye's Principles of Medicinal Chemistry, Williams and Wilkins Lippincott, $7^{\text {th }}$ Edition, 2013, 1520.

13. Suaad M. Abuskhuna, Malachy McCann, John Briody, Michael Devereux, Kevin Kavanagh, Nijhuma Kayal, Vickie McKee. Synthesis and structure of metal complexes containing zwitterionic N-hydroxyimidazole ligands, Polyhedron, 26(15), 2007, 45734580 .
14. Akagane K, Allan G G and Passot E M. Fiber surface modifications XVII, The stereotop chemistry of the adsorption of polyaza heterocycles onto cellulose, Paperija Puu, 56, 1974, 11-18.

Please cite this article in press as: Suaad M. Abuskhuna et al. Antibacterial activity of hydroxyimidazole derivatives, Asian Journal of Pharmaceutical Analysis and Medicinal Chemistry, 8(1), 2020, 1-6. 